\title{
Idiopathic chronic eosinophilic pneumonia associated with noncaseating epithelioid granulomas
}

\author{
N. Shijubo*, T. Fujishima*, S. Morita*, H. Nakata*, M. Satoh", \\ E. Uno*, T. Morikawa**, S. Abe*
}

\begin{abstract}
Idiopathic chronic eosinophilic pneumonia associated with noncaseating epithelioid grandulomas. N. Shijubo, T. Fujishima, S. Morita, H. Nakata, M. Satoh, E. Uno, T. Morikawa, S. Abe. CERS Journals Ltd 1995.

ABSTRACT: A 34 year old Japanese woman was referred to our university hospital due to pulmonary opacities and bilateral hilar lymphadenopathy on chest $\mathrm{X}$ ray. She also had uveitis, erythematous skin nodules, and oral and genital ulcers.

Laboratory data showed eosinophilia in the circulation and bronchoalveolar lavage fluid. Histological study revealed massive eosinophilic infiltration and noncaseating epithelioid granulomas in the lung and mediastinal lymph node, without evidence of vasculitis. Pulmonary opacities, lymphadenopathy, and blood eosinophilia promptly improved with corticosteroid therapy.

In this patient, idiopathic chronic eosinophilic pneumonia overlapped with features of sarcoidosis and Behçet's disease.

Eur Respir J., 1995, 8, 327-330.
\end{abstract}

\begin{abstract}
*Third Dept of Internal Medicine and Dept of Clinical Pathology, Sapporo Medical University School of Medicine, Sapporo, Japan. **Division of Thoracic Surgery, Hokkaido Keiaikai Minami Ichijo Hospital, Sapporo, Japan.
\end{abstract}

Correspondence: N. Shijubo, Third Dept of Internal Medicine, Sapporo Medical University, School of Medicine, South1, West-16, Chuo-ku, Sapporo, 060 Japan

Keywords: Behçet's disease, chronic eosinophilic pneumonia, epithelioid granuloma, sarcoidosis

Received: February 91994

Accepted after revision July 291994
Idiopathic chronic eosinophilic pneumonia (CEP) is a rare disorder characterized by striking systemic and pulmonary manifestations, blood eosinophilia, fluffy peripheral opacities on chest X-ray, and a prompt response to corticosteroid therapy [1-3]. CEP has occurred in association with several specific disorders, including scleroderma [2], ulcerative colitis [4], and some malignancies [2]. We report the case of a patient with co-existing CEP and noncaseating epithelioid granulomas, who had bilateral hilar lymphadenopathy, uveitis, erythematous skin nodules (suggesting sarcoidosis) and symptoms suggesting Behçet's disease (oral and genital ulcers).

\section{Case report}

A 34 year old Japanese housewife was hospitalized in November, 1992, due to fever and visual disturbance. She had a history of uveitis in March, 1989. Her chest X-ray had shown no abnormalities. Past medical history revealed neither asthma nor any occupational exposure to toxic fumes, dust or animals. She had smoked one pack of cigarettes daily for $16 \mathrm{yrs}$, and had taken no new drugs in the past 2 yrs. Her chest X-ray and computed tomographic (CT) scan disclosed peripheral opacities in the left upper lobe and mediastinal and hilar lymphadenopathy.

Laboratory data showed mild blood eosinophilia (leucocyte count, $8.1 \times 10^{9} \cdot l^{-1}$ with $11 \%$ eosinophils). Ophthalmological examination disclosed uveitis. Transbronchial lung biopsy was performed from the opacities; interstitial infiltration of lymphocytes was observed, but there were no specific histological findings.

Based on a clinical diagnosis of sarcoidosis, prednisolone, $30 \mathrm{mg}$ daily, was administered for the visual disturbance from December 11, 1992. The opacities, lymphadenopathy and uveitis improved remarkably. The corticosteroid dosage was gradually reduced and the therapy was stopped on February 23, 1993.

For further evaluation of her disease, the patient was referred to our university hospital in March, 1993. Examinations in the out-patient clinic over the next 3 months revealed oral and genital ulcers and erythematous skin nodules in her lower limbs. Skin biopsy was performed from an erythematous nodule; histological study showed dermoseptal panniculitis without eosinophil infiltration or epithelioid granulomas. We administered ointments, including corticosteroid for genital ulcers and erythematous skin nodules, and the lesions responded well to the medications.

Uveitis recurred in April, 1993, and local corticosteroid (eyewash) was prescribed. During the period, pulmonary opacities had wandered, and bilateral hilar lymphadenopathy and blood eosinophilia had gradually progressed. The patient was admitted in June, 1993. We had never administered any new drugs except for the ointments. No environmental evidence to suggest extrinsic allergic alveolitis was found. No cardiac murmurs or wheezes were found. Neither superficial lymph node, liver, nor spleen was palpable. No neurological abnormalities were 


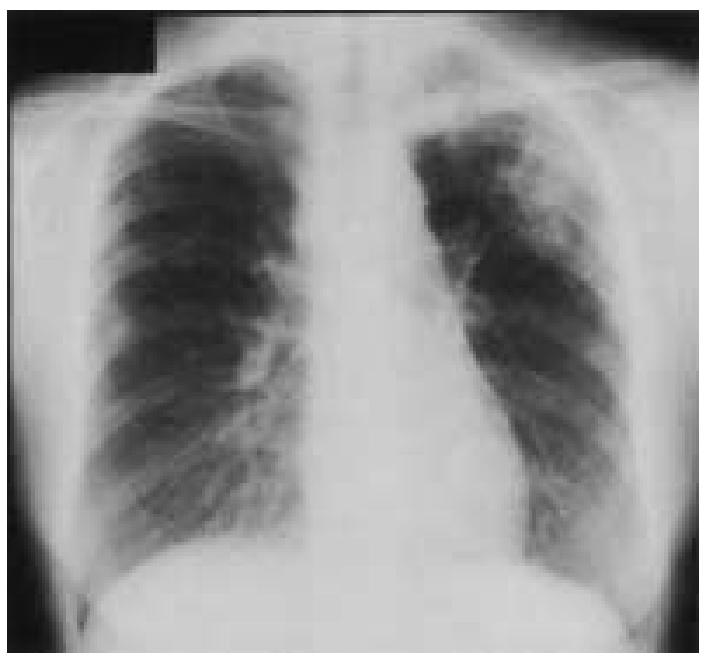

Fig. 1. - Chest X-ray on admission showing peripheral pulmonary opacities and bilateral hilar lymphadenopathy.

found. Needle test was negative. Chest X-ray revealed peripheral pulmonary opacities and mediastinal and hilar lymphadenopathy (fig. 1). High resolution CT (1.5 mm thickness) showed peripheral ground-glass opacities.

Laboratory data on admission included: leucocyte count, $12.4 \times 10^{9} \cdot l^{-1}$ with $35 \%$ eosinophils; red blood cell count, $4.58 \times 10^{12 \cdot} l^{-1}$; platelet count, $355 \times 10^{9} \cdot l^{-1}$; and normal coagulation test. Erythrocyte sedimentation rate (ESR) was $79 \mathrm{~mm} \cdot \mathrm{h}^{-1}$; serum C-reactive protein 1.27 $\mathrm{mg} \cdot \mathrm{dl}^{-1}$; and rheumatoid factor $1,687 \mathrm{IU} \cdot \mathrm{ml}^{-1}$ (normal range $<25 \mathrm{IU} \cdot \mathrm{ml}^{-1}$ ). Serum immunoglobulin levels were: immunoglobulin $\mathrm{G}$ (IgG) $23.2 \mathrm{mg} \cdot \mathrm{ml}^{-1}$; immunoglobulin A (IgA) $3.77 \mathrm{mg} \cdot \mathrm{ml}^{-1}$; immunoglobulin M (IgM) 4.25 $\mathrm{mg} \cdot \mathrm{ml}^{-1}$; and immunoglobulin $\mathrm{E}(\mathrm{IgE}), 233 \mathrm{IU} \cdot \mathrm{ml}^{-1}$ (normal range $<450 \mathrm{IU} \cdot \mathrm{ml}^{-1}$ ). Antinuclear antibody titre was $\times 80$, with a speckled pattern: anti-deoxyribonucleic acid (DNA), anti-extractable nuclear antigen (ENA), antiribonucleoprotein (RNP), and anti-Sm antibodies were negative. Results of tests for acid-fast bacilli and fungi in sputum, for parasites in stool, and for antibodies to Cryptococcus neoformans, Aspergillus fumigatus, A. niger, Micropolyspora faeni, Thernoactinomyces vulgaris, $T$. viridis, and Alternaria in serum were negative. No abnormal findings were found on examination of cardiac echogram or abdominal CT. Bone marrow aspiration examination showed an inc-reased eosinophil count, but no atypical cells (myeoblasts $0.2 \%$; promyelocytes $4.6 \%$; myelocytes $1.4 \%$; metamyelocytes $9.8 \%$; band neutrophils $17.6 \%$; segmented neutrophils $9.2 \%$; eosinophils $13.6 \%$; erythroblasts $7.8 \%$; lymphocytes $35.4 \%$; plasma cells $0.2 \%$; and monocytes $0.2 \%$ ). Bronchoalveolar lavage (BAL) of the left lingular division showed a prominently increased eosinophil count (total cell count $19.6 \times 10^{5} \cdot \mathrm{ml}^{-1}$; and differential cell count: alveolar macrophages, $1.4 \%$; lymphocytes $3.2 \%$ neutrophils $1.2 \%$; and eosinophils $94.2 \%)$.

Under thoracoscopy, biopsy was performed in the superior and anterior basal segments of the left lower lobe and the enlarged Botallo lymph node. Histological study of lung specimens revealed intra-alveolar and inters titial infiltration of eosinophils (fig. 2a), noncaseating a)

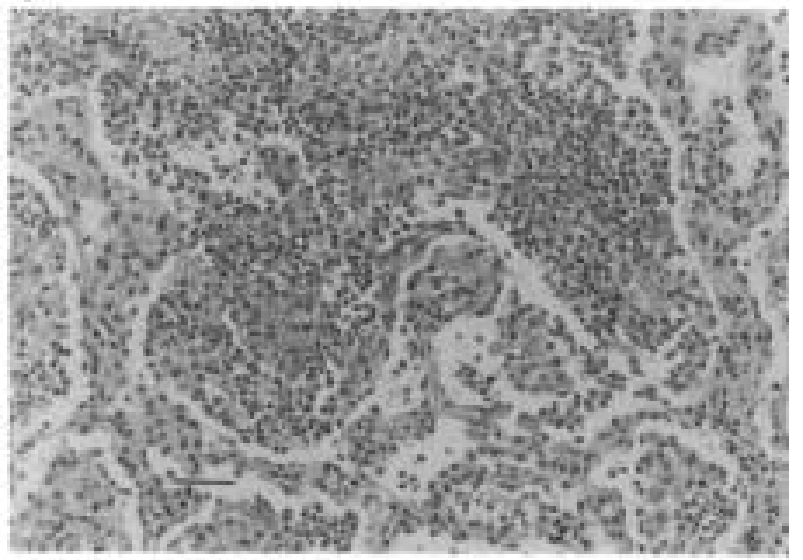

b)

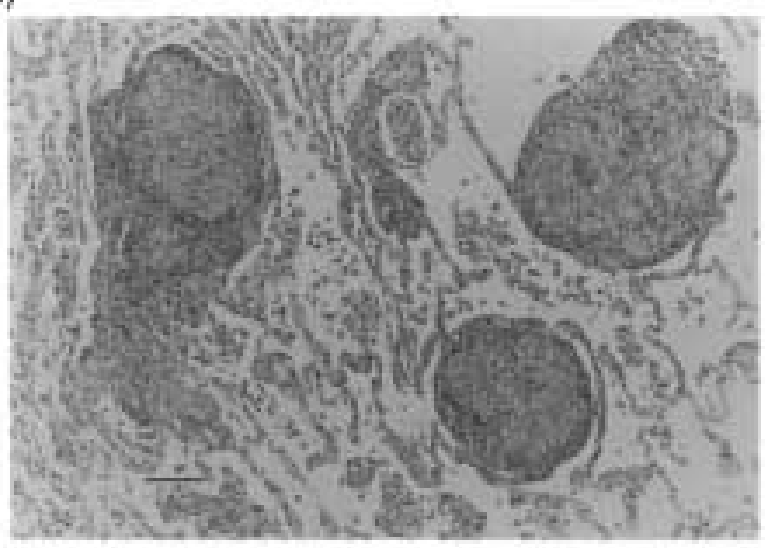

c)

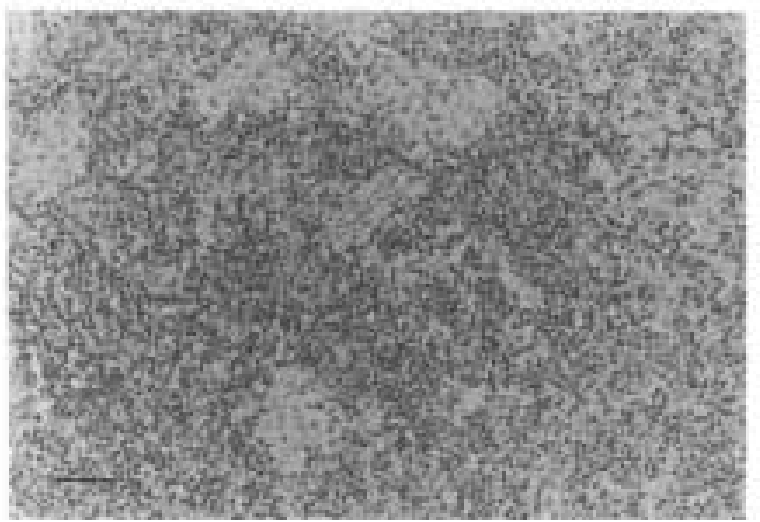

Fig. 2. - Histological study of biopsy specimens obtained from the left lung and mediastinal lymph node. a) Intra-alveolar and interstial infiltration by eosinophils observed in a lung biopsy specimen. (Scale bar= $50 \mu \mathrm{m})$. b) Noncaseating epithelioid granulomas observed in a lung biopsy specimen (Scale bar $=100 \mu \mathrm{m})$. c) Eosinophil infiltration and noncaseating epithelioid granulomas observed in a lymph node biopsy specimen. (Scale bar $=50 \mu \mathrm{m}$ ).

epithelioid granulomas (fig. 2b), and peribronchial and perivascular infiltration of lymphocytes and eosinophils. Massive eosinophilic infiltration and noncaseating epithelioid granulomas were found in the lymph node specimen (figure 2c). There was no evidence of vasculitis in these specimens, and specific staining and culture for acid-fast bacilli and fungi showed no growth.

We administered prednisolone, $30 \mathrm{mg}$ daily, from July 
12, 1993. The opacities and lymphadenopathy were promptly reduced, with a corresponding improvement in symptoms and haematological tests: leucocyte count, $12 \times 10^{9} \cdot l^{-1}$, with $2 \%$ eosinophils; ESR $7 \mathrm{~mm} \cdot \mathrm{h}^{-1}$; rheumatoid factor $92 \mathrm{IU} \cdot \mathrm{ml}^{-1}$; and antinuclear antibody negative (14 days after initiation of the therapy). The corticosteroid dosage was gradually reduced. Uveitis recurred in November, 1993, when the patient was taking $10 \mathrm{mg}$ of prednisolone daily. Local corticosteroid dosage was increased again. However, recurrence of symptoms was not observed on chest X-ray or blood examinations until May, 1994, when the patient was taking $5 \mathrm{mg}$ of prednisolone daily.

\section{Discussion}

Our patient had an unusual clinical course. In November, 1992, she had uveitis and bilateral hilar lymphadenopathy with mild blood eosinophilia. Although histological diagnosis remained unclear, these lesions responded well to corticosteroid therapy. From March, 1993, uveitis recurred, and erythematous skin nodules, oral and genital ulcers appeared. Concomitant with these symptoms, peripheral pulmonary opacities had wandered, and lymphadenopathy and blood eosinophilia had progressed. In view of available evidence, external causes of eosinophilia, such as drugs and infections, were ruled out in the present case. She was histologically confirmed to have CEP and noncaseating epithelioid granulomas in the lung and lymph node. However, it remained unclear whether noncaseating epithelioid granulomas preceded or synchronized with CEP.

We ruled out diseases having granulomas and eosinophil infiltration in the lung, especially allergic bronchopulmonary aspergillosis (ABPA) and Churg-Strauss syndrome. In our patient, tests for fungal disease were all negative, and she had no asthmatic symptoms and a normal IgE value; she was, thus, judged to be free of ABPA. Churg-Strauss syndrome is characterized by systemic necrotizing vasculitis, involving small arteries and veins, with tissue infiltration by eosinophils and extravascular granulomas $[5,6]$. The clinical hallmark of this syndrome is asthma with rhinitis, pronounced blood eosinophilia [6, 7], and systemic vasculitis.

Idiopathic CEP is a clinicopathological entity with marked systemic symptoms (fever, weight loss) and pulmonary manifestations, blood eosinophilia, peripheral opacities on chest X-ray, and a prompt response to corticosteroid therapy, as well as histological findings of intra-alveolar and interstitial infiltration by eosinophils [1-3]. CEP has been reported to be associated with several specific diseases [2, 4]. To our knowledge, there has been no report of CEP associated with sarcoidosis or extrinsic allergic alveolitis. GRANTHAM et al. [4] reported a case of CEP concomitant with ulcerative colitis. The disease activity of ulcerative colitis paralleled the disease activity of CEP. They found a prominently increased rheumatoid factor titre, which correlated with the disease activity. Our case also had a quite high rheumatoid factor titre, which seemed to be correlated with the disease activity.
Eosinophilia in BAL fluid is found in patients with sarcoidosis and extrinsic allergic alveolitis, but increases are generally moderate $(5-10 \%)[8,9]$. Our patient had bilateral hilar lymphadenopathy, uveitis, erythematous skin nodules and biopsy evidence of noncaseating epithelioid granulomas in the lung and lymph node, suggesting sarcoidosis. Extrinsic allergic alveolitis was ruled out, since she did not have any precipitating antibodies or any environmental evidence to suggest extrinsic allergic alveolitis. She had no evidence of mycobacterial, fungal, or parasitic infection, and no history of exposure to organic or inorganic materials known to cause granulomatous lung diseases. We believe that our patient has CEP concomitant with sarcoidosis: this may be clarified by her clinical manifestation in the future.

In the present case, oral and genital ulcers suggested Behçet's disease. However, oral ulcers occurred only twice, and these spontaneously improved. Uveitis, genital ulcers and erythematous skin nodules responded well to the corticosteroid treatments. No evidence of vasculitis was found in biopsy specimens.

Selective recruitment of eosinophils could be regulated by secretion of specific chemoattractants [10]. Possible candidates mediating this mechanism are interleukin-2 (IL-2) [11], interleukin-5 (IL-5) [12], and lymphocyte chemoattractant factor (LCF) [13]. IL-5 is only active on eosinophils, whilst IL-2 and LCF cause concomitant recruitment of CD4+ lymphocytes [10]. There has been increasing evidence for IL-5 being the major cytokine involved in specific eosinophilia [14-16]. IL-5 also stimulates production of autoantibodies from CD5+ Blymphocytes [17]. In the present case, although speculative, IL-5 may be concerned with eosinophil recruitment in the lung and autoantibody production, and IL-2 and/or LCF may play a role in granuloma formation and eosinophil recruitment.

\section{References}

1. Carrington $\mathrm{CB}$, Addington W, Goff AM, et al. Chronic eosinophilic pneumonia. N Engl J Med 1969; 280: 787-798.

2. Gaensler EA, Carrington CB. Peripheral opacities in chronic eosinophilic pneumonia: the photographic negative of pulmonary edema. AJR 1977; 128: 1-13.

3. Jederlinic PJ, Sicilian L, Gaensler EA. Chronic eosinophilic pneumonia: a report of 19 cases and a review of the literature. Medicine 1988; 67: 154-162.

4. Grantham JG, Meadows III JA, Gleich GJ. Chronic eosinophilic pneumonia: evidence for eosinophilic degranulation and release of major basic protein. Am J Med 1986; 80: 89-94.

5. Koss MN, Amtonovych T, Hochholzer L. Allergic granulomatosis (Churg-Strauss syndrome). Am J Surg Pathol 1981; 5: 21-28.

6. Butterfield JH, Leiferman KM. Eosinophil-associated diseases. In: Smith H, Cook RM, eds. The Handbook of Immunopharmacology: Immunopharmacology of Eosinophils. London, Academic Press, 1993: 152192.

7. Lanham JG, Elkon KB, Pusey CD, Hugues GR. Systemic vasculitis with asthma and eosinophilia: a clinical approach to the Churg-Strauss syndrome. Medicine 1984; 63: 65-81. 
8. Allen JN, Davis WB, Pacht ER. Diagnostic significance of increased bronchoalveolar lavage fluid eosinophils. Am Rev Respir Dis 1990; 142: 642-647.

9. Olivieri D, Pesci A, Bertorelli G. Eosinophilic alveolitis in immunologic interstitial lung diseases. Lung 1990; (Suppl): 964-973.

10. Kroegel C, Virchow J-C, Luttman W, Walker C, Warner JA. Pulmonary immune cells in health and disease: the eosinophil leukocyte Part I. Eur Respir J 1994; 7: 519-543.

11. Rand TH, Silberstein DS, Kornfeld H, Weller PF. Human eosinophils express functional interleukin-2 receptors. $J$ Clin Invest 1991; 88: 825-832.

12. Lopez AF, Sanderson CJ, Gamble JR, Cambell HR, Young NS, Vadas MA. Recombinant human interleukin-5 is a selective activator of human eosinophil function. J Exp Med 1988; 167: 219-224.

13. Cruikshank WW, Berman JS, Theodore AC, Bernado J, Center DM. Lymphocyte chemoattractant factor (LCF) induces CD-4 dependent intracytoplasmic signalling in lymphocytes. J Immunol 1987; 138: 3817-3823.

14. Sanderson CJ. Interleukin-5 and the regulation of eosinophil production. In: Smith $\mathrm{H}$, Cook RM, eds. The Handbook of Immunopharmacology: Immunopharmacology of Eosinophils. London, Academic Press, 1993: pp. 11-24.

15. Owen WF, Rothenberg ME, Petersen J, et al. Interleukin5 and phenotypically altered eosinophils in the blood of patients with the idiopathic hypereosinophilic syndrome. J Exp Med 1989; 170: 343-348.

16. Matsumoto R, Ando M, Kohrogi H, Araki S, Takatsu K. Interleukin-5 levels of pleural fluid and serum samples in a patients with PIE syndrome. Chest 1992; 102: 1296-1297.

17. Tominaga A, Takaki S, Koyama N, et al. Transgenic mice expressing a B-cell growth and differentiation factor gene (interleukin-5) develop eosinophilia and autoantibody production. J Exp Med 1991; 173: 429-437. 